\title{
LA OPERACIÓN PASO DEL ESTRECHO (OPE) EN CÁDIZ (ESPAÑA)
}

\author{
Manuela Mañero-Viera \\ Universidad de Cádiz \\ España
}

María Concepción Segovia-Cuevas

Universidad de Cádiz

España 
Panorama Económico, Vol. 25 - No. 3 (Julio - Septiembre de 2017), pp. 381-398

Manuela Mañero-Viera
María Concepción Segovia-Cuevas

La Operación Paso del Estrecho (OPE) en Cádiz (España)

\begin{abstract}
Resumen
En los años 70 y 80 se produjo un éxodo de mano de obra magrebí hacia algunos países de la Unión Europea (Francia, Alemania, Holanda, entre otros), iniciándose el movimiento migratorio denominado Paso del Estrecho. Este consiste en el traslado una vez al año por parte de esta población a sus países de origen con fines de visita familiar. Los puertos españoles, en especial, los del Estrecho de Gibraltar colpasaron, por lo que el Gobierno Español decidió organizar y planificar el tránsito de personas, mediante la Operación Paso del Estrecho (OPE). Más de dos millones y medio de pasajeros y vehículos atraviesan Europa hasta España con el objetivo de cruzar a África, principalmente a traves de los Puertos de la Bahía de Algeciras (Algeciras y Tarifa), los cuales concentran el 75\% del flujo migratorio. En el municipio de Tarifa (de aproximadamente 18.000 habitantes), con características marcadas por ser un punto históricamente geoestratégico, se produce un incremento sustancial de la población durante los el desarrollo de la OPE. Este hecho hace necesario que se plantee el estudio del caso de este municipio, debido a las carencias en infraestructura que posee.
\end{abstract}

Palabras clave: Operación Paso del Estrecho, flujo migratorio, población magrebí, Tarifa, infraestructuras.

Clasificación JEL: F22, R23, F29, R20, R29

\title{
The passage of Gibraltar's Strait in Tarifa (Cádiz, Spain)
}

\begin{abstract}
In the 70s and 80s, there was an exodus of Maghreb labor to some countries of the European Union (France, Germany, Holland, among others), which launched the migratory movement called Paso del Estrecho. This is the transfer made during the summer of members of this active population to their country of origin for family purposes. This caused the collapse of passengers in Spanish ports, especially those in the Strait of Gibraltar. The Spanish Government has therefore decided to organize and plan this transit of people, known as Operation Paso del Estrecho (OPE). More than two and a half million passengers and vehicles travel across Europe to Spain to visit Africa. The ports of Algeciras Bay (Algeciras and Tarifa) account for $75 \%$ of migration flows. In the municipality of Tarifa (population around 18,000), whose characteristics are characterized by a historical geostrategic point, there is an exponential increase in the population during the months of the OPE. This fact implies that the study of the case of this municipality considers, because of the insufficiencies of infrastructures at its disposal.
\end{abstract}

Keywords: Operation Paso del Estrecho, migration, Maghrebi population, rate, infrastructure.

JEL Classification: F22, R23, F29, R20, R29

\section{Le passage du détroit de Gibraltar à Tarifa (Cadix, Espagne)}

\section{Résumé}

Dans les années 70 et 80, il y avait un exode de la main-d'œuvre maghrébine vers certains pays de l'Union européenne (la France, l'Allemagne et la Hollande, entre autres), ce qui a lancé le mouvement migratoire appelé Paso del Estrecho. Il s'agit du transfert effectué pendant l'été des membres de cette population active vers leur pays d'origine afin de rendre visite à leurs proches. Cela a provoqué l'effondrement des passagers dans les ports espagnols, en particulier ceux du détroit de Gibraltar. Le gouvernement espagnol a donc décidé d'organiser et de planifier ce transit de personnes, connu sous le nom d'Opération Paso del Estrecho (OPE). Plus de deux millions et demi de passagers et de véhicules parcourent l'Europe à travers l'Espagne pour se rendre en Afrique. Les ports de la baie d'Algésiras (Algeciras et Tarifa) concentrent 75\% des flux migratoires. Dans la municipalité de Tarifa (d'environ 18000 habitants), dont les caractéristiques sont caractérisées par un point de géostratégie historique, il existe une augmentation exponentielle de la population au cours des mois de l'OPE. Ce fait entraîne que l'étude du cas de cette municipalité considère, en raison des insuffisances d'infrastructures à sa disposition.

Mots-clés: Opération Paso del Estrecho, flux migratoire, population maghrébine, tarif, infrastructure.

Nomenclature JEL: F22, R23, F29, R20, R29 


\section{La Operación Paso del Estrecho (OPE) en Cádiz (España)}

\section{INTRODUCCIÓN}

El presente trabajo tiene como objetivo identificar cómo afecta la Operación Paso del Estrecho (OPE) al municipio de Tarifa (Cádiz, España). En este artículo, se realiza una presentación del municipio profundizando en las características que le convierten en un punto geoestratégico para la Unión Europea. Asimismo, se ha estudiado la evolución de la OPE desde 2013 y la influencia de esta en un territorio que ve incrementado el tráfico de personas y pasajeros de forma considerable en los meses de junio a septiembre. Este movimiento migratorio que cada año se repite en dicho periodo desde los años 80 del pasado siglo, se ha ido convirtiendo en el segundo movimiento de migración más importante del mundo islámico, después de la peregrinación a la Meca.

Se desea conocer las posibles implicaciones que tiene una operación de esta envergadura -más de un millón y medio de pasajeros y de 200,000 automóviles al año- para un municipio, cuyo núcleo poblacional central, donde se ubica el puerto de entrada y salida, tiene aproximadamente 13.000 habitantes. Se trata de identificar los posibles beneficios para la actividad económica municipal y, también, las tensiones y problemas que genera dadas las limitaciones en infraestructuras y servicios de la localidad.

\section{EL MUNICIPIO DE TARIFA Y SUS CARACTERÍSTICAS}

La Constitución Española de 1978, en su artículo 137, organiza territorialmente al Estado español en municipios, provincias y Comunidades Autónomas. En la actualidad,

\footnotetext{
* Autor para correspondencia

Correos electrónicos: manuela.maneroviera@aluum.uca.es*, concepcion.segovia@uca.es
} 
Figura 1. Posición del municipio Tarifa

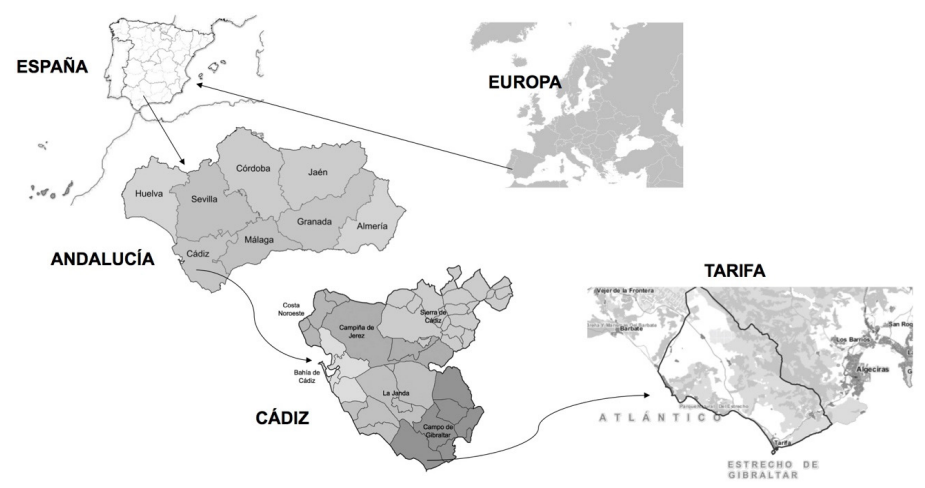

Fuente: elaboración propia.

España está dividida en 17 Comunidades Autónomas y dos ciudades autónomas, 50 provincias y 8.117 municipios. En su artículo 140, la Constitución establece que los municipios poseen personalidad jurídica plena, correspondiendo su gobierno y administración a sus respectivos ayuntamientos, integrados por el alcalde y los concejales. Las haciendas locales disponen de los medios para el desempeño de las funciones que la ley les atribuye, provenientes de tributos propios y de las asignaciones del Gobierno Central y de las Comunidades Autónomas (artículo 142). Existen otras entidades locales territoriales, tales como las comarcas $^{1}$, las áreas metropolitanas ${ }^{2}$ y las mancomunidades de municipios ${ }^{3}$,

1 Una comarca es una entidad local territorial que agrupa varios municipios, con características geográficas, históricas y socioeconómicas comunes, cuya creación se regula en el art. 42 de la Ley Reguladora de las Bases de Régimen Local.

2 "Las áreas metropolitanas son entidades locales integradas por los municipios de grandes aglomeraciones urbanas entre cuyos núcleos de población existen vinculaciones económicas y sociales que hagan necesaria la planificación conjunta y coordinación de determinados servicios y obras". (Ley Reguladora de las Bases de Régimen local, art. 43.2).

3 La mancomunidad es una asociación voluntaria de municipios para la ejecución en común de obras y servicios, con competencias y recursos cedidos por los municipios socios (Ley Reguladora de las Bases de Régimen local, art. 44). reguladas por la Ley Reguladora de las Bases de Régimen Local.

Tarifa es un municipio de la provincia de Cádiz (una de las ocho provincias que forman la Comunidad Autónoma de Andalucía), perteneciente a la Comarca y Mancomunidad del Campo de Gibraltar (Figura 1). Tiene una extensión territorial de $420 \mathrm{~km}^{2}$ y es un municipio multipolar, con un núcleo central de población (Tarifa) que concentra $80 \%$ de la población municipal, dos núcleos de tamaño medio (Facinas y Tahivilla) y 19 núcleos más pequeños, entre los que destacan El Almarchal, La Zarzuela, Atlanterra, Bujeo y Bolonia.

La posición de Tarifa tiene carácter geoestratégico, pues está situada sobre el Estrecho de Gibraltar que es el punto de paso entre Europa y África $(14 \mathrm{~km}$ entre los puntos más próximos) y entre el Océano Atlántico y el Mar Mediterráneo (eje América-Asia), siendo el punto más meridional de Europa y formando parte de la frontera sur de la Unión Europea (figura 1). Cada año, alrededor de 150.000 buques transitan el Estrecho (Informe Anual Salvamento Marítimo de Tarifa, 2015), siendo este el segundo más transitado del mundo, después del English Channel. 
En enero de 2016, el municipio contaba con 18.116 habitantes (Padrón municipal -INEBase) con una densidad de población de $42 \mathrm{hab} . / \mathrm{km}^{2}$, cifra inferior a la provincial, 166 hab. $/ \mathrm{km}^{2}$, y a la regional, 96 hab. $/ \mathrm{km}^{2}$. Cabe destacar que, en los últimos años, Tarifa ha experimentado un aumento de su población censada como consecuencia de la afluencia de población extranjera, principalmente italiana, alemana y holandesa.

Se tienen indicios de asentamientos en el municipio tarifeño desde muy antiguo, como las pinturas rupestres de la Cueva del Moro ${ }^{4}$, correspondiente al periodo paleolítico y la Necrópolis de los Algarbes ${ }^{5}$, perteneciente a la edad de los metales. Sin embargo, el yacimiento arqueológico más importante del municipio son las ruinas de la ciudad de Baelo Claudia, ciudad romana edificada en el siglo II a. C. situada en Bolonia.

El municipio de Tarifa, en el año 710, fue el punto deentrada de las tropas musulmanas de Tarif Abu Zara, el comandante de Tarik ben Ziyad a la Península Ibérica ${ }^{6}$ en donde instalaron una fortificación militar, ayudando a que Tarik invadiera el monte Calpe $^{7} \mathrm{y}$, posteriormente, se extendieran por el territorio peninsular.

4 La Cueva del Moro fue descubierta por el espeleólogo Lothar Bergman en 1994. Este yacimiento está considerado como el arte rupestre más antiguo de la provincia de Cádiz del Paleolítico Superior con una antigüedad de más de 20.000 años.

5 La Necrópolis de los Algarbes fue descubierta como tal en 1967 por Carlos Posac Mon, fue usada como lugar de enterramiento y de culto para distintas civilizaciones como los fenicios y los romanos entre otras. Se considera el complejo arqueológico más importante de la provincia de Cádiz correspondiente a la edad de los metales.

6 Las tropas musulmanas desembarcaron en la Isla de las Palomas. Esta isla es conocida como Punta de Tarifa o punta marroquí, está unida al municipio por un istmo artificial que sirve como división entre el océano Atlántico y el mar Mediterráneo.

7 En la actualidad, se conoce como el Peñón de Gibraltar y en él se encuentra la colonia inglesa de Gibraltar.
La ocupación musulmana duró hasta 1340, cuando las tropas castellanas y portuguesas de Alfonso XI recuperaron Tarifa para Castilla, tras años de batallas. Durante los siglos siguientes y ya en época moderna, el municipio se va consolidando como tal, dedicándose su población fundamentalmente a la pesca, la ganadería y algunos cultivos. En 1704, Tarifa se convierte en zona de operaciones militares a raíz de la pérdida de España del Peñón de Gibraltar ${ }^{8}$. Posteriormente, sufrió las consecuencias de la invasión napoleónica $\mathrm{y}$ de las sucesivas crisis económicas que jalonaron los siglos XIX y XX en España. Desde la guerra civil (1936-1939) y la dictadura de Franco (1939-1975), por su posición geoestratégica, tuvo una fuerte presencia militar que ha configurado parte de su estructura económica reciente.

El municipio tarifeño tiene aproximadamente el $60 \%$ de su territorio protegido por dos parques naturales: el del Estrecho y el de los Alcornocales. El Parque Natural del Estrecho, situado entre los municipios de Tarifa y Algeciras, posee una elevada diversidad de especies marinas y terrestres, constituyendo una zona de elevado de interés biológico y biogeográfico. Bajo esta protección de parque natural se hallan también las playas de Los Lances?, Valdevaqueros, conocida mundialmente por los deportes acuáticos (windsurf y kitesurf) y Bolonia ${ }^{10}$, con una duna de más de 30 metros de altura y 200 metros de anchura. El Parque Natural de los Alcor-

\footnotetext{
8 España cedió el Peñón de Gibraltar a Gran Bretaña en 1713 en el Tratado de Utrecht, para que la corona británica reconociera al rey Felipe V.

9 La playa de los Lances se encuentra protegida por la figura de Paraje Natural. Este fue declarado el $28 \mathrm{de}$ julio de 1989 por la Consejería de Medio Ambiente y Ordenación del Territorio de la Junta de Andalucía.
}

10 La duna de Bolonia está protegida bajo la consideración de monumento natural, fue declarada como tal el 23 de noviembre de 2001. 
nocales agrupa varias sierras, dándose en ellas el mayor bosque de alcornoques de la Península Ibérica, con una vegetación y fauna muy diversa. El 50\% de su superficie está dedicada a la extracción de corcho de los alcornoques. Estos espacios protegidos incluyen la sierra de la Plata, la Loma de San Bartolomé, la sierra de Enmedio y la sierra del Cabrito. Estas se ubican de Norte a Sur y se caracterizan por encontrarse cerca de la costa y por poseer arte rupestre en ellas.

El clima del municipio es mediterráneo, con lluvias más frecuentes en invierno, y se caracteriza por sus vientos de levante y de poniente. El viento de levante procede del Mar de Alborán y del Nordeste de África, trae temperaturas altas $\mathrm{y}$ aire seco. Y el viento de poniente proviene del Océano Atlántico y trae temperaturas frescas y húmedas.

Tradicionalmente, el municipio ha basado su economía en la pesca. Desde la época romana la economía de este municipio se ha enfocado hacia las conservas, las salazones y el atún. En la actualidad, la pesca no tiene la importancia de los siglos anteriores; sin embargo, sigue siendo un sector económico importante para el municipio, principalmente las capturas del atún rojo y del voraz. Para la captura del atún se sigue utilizando el método tradicional de la almadraba ${ }^{11}$ y están limitadas en tiempo y cantidad por las regulaciones de la Unión Europea. La pesca del voraz (besugo de la pinta) es la principal

11 La almadraba es una forma tradicional de pesca de atunes; se tiene constancia de ella desde tiempos prerromanos y sigue utilizándose en la actualidad. Consiste en una jaula de redes (unida por dos barcos) donde los atunes caen en su migración hacia el Mediterráneo; los pescadores examinan uno por uno cada atún para elegir las piezas óptimas. Al proceso de extracción de estas piezas se le denomina "levantá". Una gran parte de las capturas son compradas por barcos asiáticos, fundamentalmente japoneses. producción de los barcos tarifeños durante todo el año, excepto durante las paradas biológicas. Las producciones agrarias más significativas de este municipio son el girasol, el trigo y la ganadería vacuna de raza retinta ${ }^{12}$.

La principal industria tarifeña es la producción de energía eólica. Tarifa cuenta con dos parques eólicos, uno situado en la sierra de la Plata (entre Facinas y Tahivilla) y el otro en el Bujeo. De los 2.135 aerogeneradores que hay en la Comunidad Autónoma de Andalucía, el $26,3 \%$ se encuentran en este municipio y producen 5.622,3 Mw, según la Asociación Empresarial Eólica. El resto de la actividad industrial del municipio se concentra en dos fábricas, una conservera y otra de harinas refinadas y aceites de girasol para alimentación animal. Además, hay algunas panificadoras artesanas y otras pequeñas empresas de alimentación y de confección de artesanías artísticas y ornamentales. La crisis económica ha afectado fuertemente al sector de la construcción que, tras años dedicados a la edificación de apartamentos turísticos, se ha reconvertido a la construcción de viviendas familiares y hoteles y a la rehabilitación del centro histórico.

El municipio de Tarifa posee un gasoducto y dos conexiones eléctricas submarinas que transportan gas natural y electricidad, respectivamente, entre España y Marruecos $^{13}$. En cuanto a la red viaria, el municipio se encuentra conectado con la provincia por la carretera nacional N-340; además, existe una red de carreteras secundarias y caminos rurales que

$12 \mathrm{El}$ retinto es una raza de ganado vacuno de carne autóctona de Andalucía Occidental y Extremadura.

13 La primera conexión eléctrica submarina en Tarifa se instaló en 1997 (400kW) y la segunda en 2006 (400kW). El gasoducto del Magreb entra en España por Tarifa y transporta gas natural procedente de Argelia. 
conectan los núcleos de población que no se encuentran a lo largo de la N-340.

En los últimos 35 años, Tarifa se ha posicionado como uno de los municipios con más potencial turístico de la provincia de Cádiz. Esto se debe principalmente al turismo de sol y playa y al turismo de deportes acuáticos (windsurf y kitesurf). La ocupación hotelera refleja la situación en la que se encuentra el turismo tarifeño con un $100 \%$ de ocupación durante los meses de verano. La afluencia de personas se ha visto incrementada también, por la diversificación de la oferta de actividades turísticas durante el resto del año, como son los espectáculos en el teatro romano de Baelo Claudia, las rutas de senderismo por los parques naturales y el avistamiento de aves y cetáceos.

\section{OPERACIÓN PASO DEL ESTRECHO (OPE)}

Tarifa se ha convertido en un lugar de paso para muchos viajeros que cruzan hacia África o Europa, especialmente durante la época que dura la Operación Paso del Estrecho (OPE). El puerto de Tarifa recoge anualmente más de un millón de pasajeros en su línea Tarifa-Tánger-Ville(Marruecos)Tarifa. Está gestionado por la Autoridad Portuaria Bahía de Algeciras (APBA), que engloba a todos los puertos del Campo de Gibraltar, excepto el de la colonia británica.

La OPE surge para dar respuesta a las demandas de la población, principalmente de Algeciras, que se quejaban cada verano de la difícil situación que se vivía con la llegada masiva de viajeros. La insalubridad, invasión de espacios públicos, el colapso portuario, etc., hicieron que las autoridades locales plantearan la "Operación Avalancha" que estuvo operativa para el municipio de Algeciras entre los años 1983 a 1985 (Finotelli, 2007;
Gómez Vallecillo, 2002). Durante estos años, el gobierno español y la embajada de Marruecos en España empezaron a establecer relaciones de colaboración con el fin de gestionar de manera más eficiente y segura el flujo de personas y vehículos (Fernández Alles \& Fernández Alles, 2015).

En 1986, el tránsito migratorio por vacaciones se masificó a causa de la exigencia de Francia de pedir visados a los magrebíes que visitaban a sus familiares (Acosta Sánchez, 2016; Heering, van der Erf, \& van Wissen, 2004; StreiffFénart \& Poutignat, 2008). Se decidió entonces que el Ministerio del Interior de España por medio de la Dirección General de Protección Civil se encargara de organizar y planificar el tránsito de pasajeros, creando los planes especiales de la "Operación tránsito" hasta 1991. En 1987 se puso en marcha el primer plan centrado, fundamentalmente, en labores humanitarias y sanitarias, así como en la adecuación de los puertos y zonas de descanso para los pasajeros. Todos estos planes han servido de aprendizaje para paliar los problemas detectados, con objeto de mejorar la seguridad, organización e infraestructuras de los sitios afectados. Entre las actuaciones de estos planes destacan las inversiones en adecuación de infraestructuras y recursos humanos, aumento de la oferta naviera, etc. (Fernández Alles \& Fernández Alles, 2015)

A partir de 1991, estas operaciones anuales pasaron a denominarse Operación Paso del Estrecho. La OPE se define como un plan especial de la Dirección General de Protección Civily Emergencias, gestionado por el Ministerio del Interior de España cuyo fin principal es coordinar, en materia de seguridad, asistencia y transporte, la afluencia de inmigrantes magrebíes que cruzan el Estrecho de Gibraltar durante 
las vacaciones de verano (Protección civil, Ministerio del Interior, 2016). Este plan logístico aglutina a localidades de seis Comunidades Autónomas (Informe de verano del Ministerio del Interior, 2016):

- Irún (Guipúzcoa) en el País Vasco como puesto fronterizo

- La Junquera (Gerona), como puesto fronterizo y Barcelona como área de descanso) en Cataluña.

- Madrid (C.A. de Madrid) como área de descanso y puesto de información.

- Valdepeñas en Castilla la Mancha, como área de descanso.

- Alicante (Comunidad Valenciana) como puerto de entrada/salida y paso fronterizo.

- Granada como área de descanso y Almería, Málaga, Motril (Granada), Algeciras (Cádiz) y Tarifa (Cádiz) en
Andalucía, como puertos de entrada/ salida y pasos fronterizos.

- Las ciudades autónomas de Ceuta y Melilla como pasos fronterizos y puertos de entrada/salida.

Los viajeros con sus vehículos vienen fundamentalmente de Francia, Bélgica, Alemania y Holanda (Heering et al., 2004; Ouali, 2003; Dirección General de Protección Civil, 2017). La OPE tiene planificadas distintas rutas e itinerarios (figura 2) para los magrebíes que entran en territorio español desde o hacia el resto de Europa, guiándolos de frontera a frontera, ofreciéndoles información y ayuda constante. Estas rutas nacen en los pasos fronterizos y están provistas de puntos de ayuda, de descanso, de información sobre tráfico y carreteras, etc.

\section{Figura 2. Rutas e itinerarios OPE}

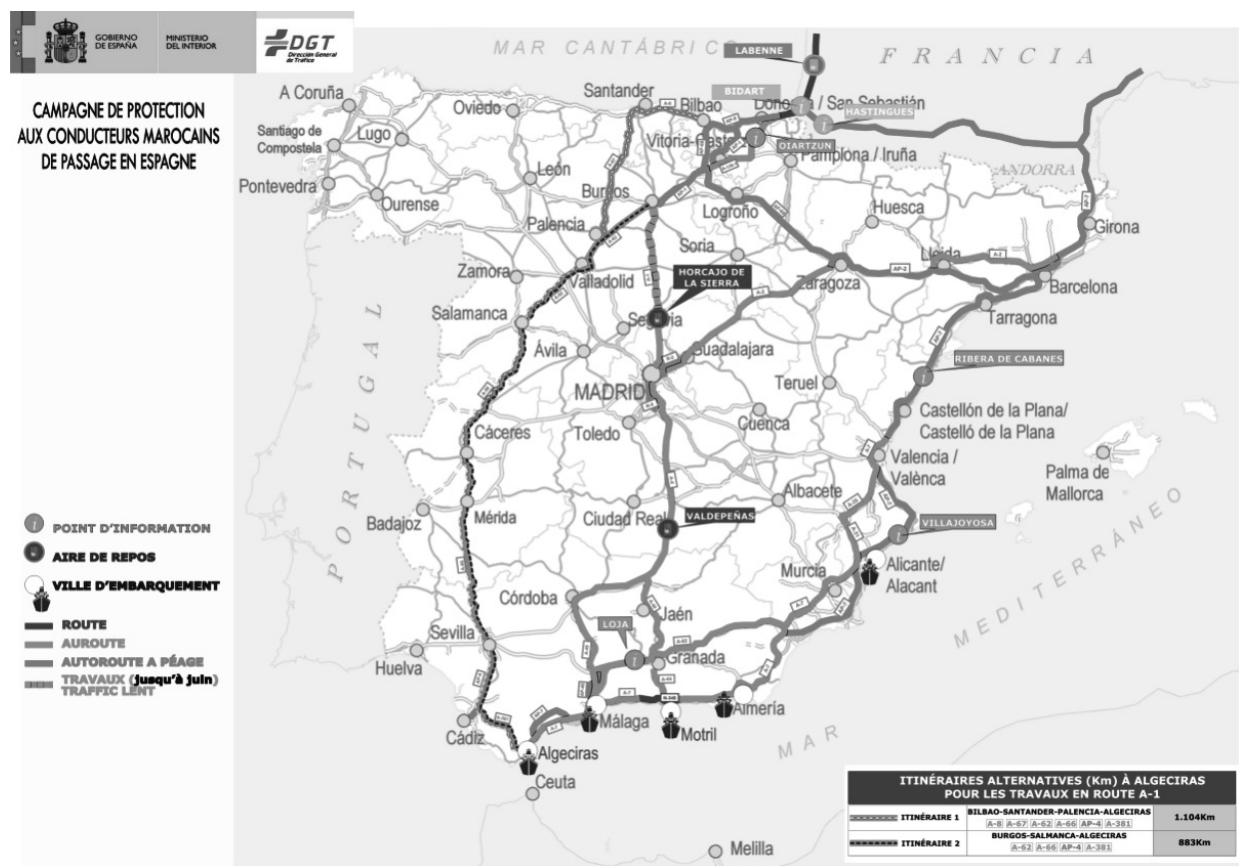

Fuente: Dirección General de Protección Civil de España 
La OPE atiende a un flujo migratorio de más de 2,5 millones de personas magrebíes que viajan por Europa hasta llegar a las costas españolas y cruzan el Estrecho de Gibraltar hacia Marruecos y Argelia, principalmente, con el fin de visitar a su familia. Está planificada en dos fases: la salida y el retorno. La fase de salida abarca desde el 15 de junio hasta el 15 de agosto, y la fase de retorno comprende desde el 15 de julio hasta el 15 de septiembre (Dirección General de Protección Civil). Los principales puertos que se ven afectados por la OPE en la fase de salida son Algeciras, Tarifa y Almería; y en la fase de retorno, Melilla y Ceuta. El tránsito de viajeros por los puertos gestionados por la Autoridad Portuaria Bahía de Algeciras en 2016 (Algeciras y Tarifa), superó el 75\% del total de la OPE.
El tráfico de pasajeros implicados en la OPE en los últimos tres años ha ido incrementándose (tabla 1), llegando en 2016 a un aumento del 12,2\% respecto a 2014. El número de vehículos (tabla 2) también ha aumentado en ese periodo, aunque no en la misma medida (10,1\%). A pesar que, en conjunto, el número de pasajeros y de vehículos ha aumentado todos los años, este aumento no se ha distribuido proporcionalmente entre los puertos involucrados; algunos de ellos incluso han tenido una disminución del tráfico de personas y vehículos en el periodo considerado debido a distintos motivos, como la situación climatológica o la elección de otras rutas y puertos de España.

Tabla 1. Movimiento de pasajeros en la OPE (ida y retorno), 2014-2016.

\begin{tabular}{|c|c|c|c|c|}
\hline & Puerto & 2014 & 2015 & 2016 \\
\hline \multirow{7}{*}{$\begin{array}{c}\text { Fase de salida } \\
\text { (15 junio- } \\
15 \text { agosto) }\end{array}$} & Motril & 121.009 & 133.484 & 115.300 \\
\hline & Málaga & 25.254 & 35.324 & 35.513 \\
\hline & Almería & 163.919 & 173.473 & 213.179 \\
\hline & Alicante & 36.582 & 37.858 & 32.945 \\
\hline & Algeciras & 699.810 & 746.715 & 826.085 \\
\hline & Tarifa & 186.045 & 213.526 & 190.071 \\
\hline & TOTAL & 1.232 .619 & 1.340 .380 & 1.413 .093 \\
\hline \multirow{9}{*}{$\begin{array}{c}\text { Fase de retorno } \\
\text { (15 julio-15 } \\
\text { septiembre) }\end{array}$} & Alhucemas & 17.357 & 18.325 & 13.444 \\
\hline & Argel & 3.387 & 15.552 & 10.974 \\
\hline & Ceuta & 283.357 & 277.086 & 260.266 \\
\hline & Ghazaouet & 15.987 & 17.357 & 13.527 \\
\hline & Melilla & 138.958 & 178.373 & 177.465 \\
\hline & Orán & 54.721 & 51.203 & 43.798 \\
\hline & Nador & 112.975 & 110.356 & 126.704 \\
\hline & Tánger & 689.403 & 804.319 & 801.704 \\
\hline & TOTAL & 1.316.145 & 1.472 .571 & 1.447 .882 \\
\hline TOTAL & & 2.548 .764 & 2.812 .951 & 2.860 .975 \\
\hline
\end{tabular}

Fuente: Elaboración propia desde Dirección General de Protección Civil de España. 
Tabla 2. Movimiento de automóviles en la OPE (ida y retorno), 2014-2016.

\begin{tabular}{|c|c|r|r|r|}
\hline & Puerto & 2014 & 2015 & 2016 \\
\hline \multirow{4}{*}{$\begin{array}{c}\text { Fase de salida } \\
\text { (15 junio- } \\
15 \text { agosto) }\end{array}$} & Motril & 28.374 & 32.211 & 28282 \\
\cline { 2 - 5 } & Málaga & 3.535 & 5.583 & 5.217 \\
\cline { 2 - 5 } & Almería & 34.879 & 37.112 & 46.225 \\
\cline { 2 - 5 } & Alicante & 9.913 & 9.918 & 8.785 \\
\cline { 2 - 5 } & Algeciras & 190.143 & 187.857 & 213.312 \\
\cline { 2 - 5 } & Tarifa & 31.659 & 37.332 & 30.962 \\
\hline \multirow{4}{*}{$\begin{array}{c}\text { Fase de Retorno (15 } \\
\text { julio-15 septiembre) }\end{array}$} & TOTAL & 298.503 & 272.681 & 301.821 \\
\cline { 2 - 5 } & Alhucemas & 3.880 & 4.124 & 3.029 \\
\cline { 2 - 5 } & Argel & 682 & 3.404 & 2.420 \\
\cline { 2 - 5 } & Ceuta & 60.383 & 58.414 & 55.529 \\
\cline { 2 - 5 } & Melilla & 3.658 & 3.818 & 3.276 \\
\cline { 2 - 5 } & Orán & 28.428 & 37.673 & 37.560 \\
\cline { 2 - 5 } & Nador & 12.978 & 11.663 & 10.194 \\
\cline { 2 - 5 } & Tánger & 21.800 & 20.458 & 24.947 \\
\cline { 2 - 5 } & TOTAL & 164.654 & 178.670 & 185.136 \\
\hline & & 296.463 & 318.224 & 322.091 \\
\hline TOTAL & 594.966 & 590.905 & 623.912 \\
\hline
\end{tabular}

Fuente: Elaboración propia desde Dirección General de Protección Civil de España.

Este incremento del tráfico de la OPE ha generado un aumento del número de acciones de asistencia sanitaria realizadas cada año, principalmente, atendiendo afecciones cutáneas, mareos y cefaleas. Otro tanto ha ocurrido con las ayudas sociales centradas en el suministro de información en distintas lenguas y la resolución de problemas con la documentación. En 2016, a pesar del incremento del número de pasajeros, las acciones de asistencia sanitaria y social han disminuido en un 1,0\% y un 8,3\% respecto a 2014 (Tabla 3).

Tabla 3. Asistencias sanitarias y sociales, OPE 2016

\begin{tabular}{|c|r|r|}
\hline Autoridades Portuarias & Asistencias sanitarias & \multicolumn{1}{|c|}{ Asistencias sociales } \\
\hline Bahía de Algeciras & 526 & 1.532 \\
\hline Alicante & 156 & 477 \\
\hline Almería & 1.000 & 926 \\
\hline Málaga & 66 & 910 \\
\hline Motril & 207 & 85 \\
\hline Ceuta & 218 & 681 \\
\hline Melilla & 360 & 38 \\
\hline TOTAL & 2.533 & 4.649 \\
\hline
\end{tabular}

Fuente: Ministerio del Interior 
Este dispositivo migratorio se rige por dos principios, el principio de la intercambiabilidad $y$ el principio de la libre competencia. El principio de intercambiabilidad consiste en que la OPE coordina con las compañías navieras las distintas rutas y prevé la posibilidad de intercambiar los billetes entre las distintas compañías, en momentos de gran aglomeración de pasajeros y vehículos en determinadas fechas, como navidades o verano (Fernández Alles \& Fernández Alles, 2015). Hasta el momento, sólo se ha tenido que recurrir a la aplicación de este principio en el verano de 2008, así como en algún día específico en años posteriores (después de un temporal que impide el tráfico durante unos días). El principio de libre competencia proclama la existencia de libre elección tanto para el pasajero como para las navieras; sin embargo, algunas de las principales compañías fueron sancionadas por acordar de forma ilegal precios, horarios, condiciones comerciales, etc. en 2011 (Resolución de la Comisión Nacional de la Competencia, 2011). Se estableció entonces que la CNC durante la OPE vigilaría el funcionamiento de las navieras para salvaguardar los derechos de los viajeros y así contribuir a la gestión y buen funcionamiento de la operación.

Cabe señalar que, además de este periodo vacacional, existe un incremento considerable del tráfico de magrebíes por el Estrecho con motivo de algunas fiestas señaladas del calendario musulmán (Tabla 4) y para el que se activa un operativo semejante a la OPE, aunque en menor escala.

\section{OPERACIÓN PASO DEL ESTRECHO EN TARIFA}

El puerto de Tarifa está gestionado por la Autoridad Portuaria Bahía de Algeciras (APBA) y se dedica especialmente al tráfico de pasajeros y vehículos. Está dividido en varias zonas: zona de embarque y atraque del ferry, muelle pesquero y lonja y puerto deportivo y recreativo Las compañías navieras que operan en el municipio son Förde Reedorei Seetouristik Iberia (FRS) e Inter-Shipping SARL. Durante la OPE, cuando se producen situaciones de congestión, otras compañías navieras se incorporan al tráfico del Estrecho en este puerto (Balearia, Transmediterránea, etc.).

La Autoridad Portuaria Bahía de Algeciras (APBA) gestiona el $75 \%$ de la OPE, asignándole un presupuesto de un millón y medio de euros (Informe Económico APBA, 2016). Estos fondos se emplean para la contratación de más de 570 personas entre auxiliares y técnicos de información, así como para la adquisición de nuevas tecnologías para la seguridad (el software Hércules, que recoge permanentemente la ocupación de los puertos de Algeciras y Tarifa y las 475 cámaras del sistema captación y tratamiento de imágenes) y para la gestión portuaria (Brain Port Analitics, destinada a facilitar la información a los usuarios por medio de una app). Además, durante el resto del año, la APBA realiza diversas inversiones en infraestructuras con objeto de mejorar las condiciones de sus puertos para la OPE. En 2016, la autoridad portuaria destinó cuatro

Tabla 4. Principales festividades calendario musulmán.

\begin{tabular}{|c|c|c|c|c|}
\hline & 2013 & 2014 & 2015 & 2016 \\
\hline Inicio del Ramadán & 9 junio & 28 junio & 18 junio & 6 junio \\
\hline Fin del Ramadán (Eid Al-Fitr) & 8 agosto & 28 julio & 17 julio & 6 julio \\
\hline Eid Al-Adha (Fiesta del Cordero) & 15 octubre & 4 octubre & 24 septiembre & 11 septiembre \\
\hline
\end{tabular}

Fuente: Elaboración propia, El Crisol blog. 
millones y medios de euros a la ejecución de la glorieta de entrada al Puerto de Tarifa, la reordenación del área de pre-embarque, la instalación de un serpentín en el área de pasajeros de la estación marítima de Tarifa y la pavimentación y balizamiento del puerto (Informe Anual APBA, 2016).

Los buques alojados en el Puerto de Tarifa realizan una única ruta, Tarifa-Tánger Ville-Tarifa. Esta se encuentra operativa durante todo el año, exceptuando los días de fuertes vientos que implican el cierre del puerto. En el periodo 2013-2016, el tráfico anual de pasajeros y vehículos particulares en la ruta Tarifa-Tánger VilleTarifa ha disminuido en un 4,4\% y un $13,2 \%$, respectivamente (tabla 5). Es muy posible que, tras el máximo alcanzado en 2013 , los usuarios hayan elegido otros puertos debido al nivel de congestión alcanzado en el puerto de Tarifa, trasladando este problema a los puertos alternativos (principalmente Algeciras). Al parecer se estaría produciendo un movimiento pendular que se empieza a notar en 2016 a pesar que, en este último año, se han producido un mayor número de incidencias que han mantenido el puerto cerrado que en los años anteriores (Informes diarios de situación, APBA).

\section{Tabla 5. Número de viajeros y vehículos en la ruta Tarifa-Tánger Ville-Tarifa, 2013-2016}

\begin{tabular}{|c|c|c|}
\hline & Viajeros & Vehículos \\
\hline $\mathbf{2 0 1 3}$ & 1.462 .093 & 270.124 \\
\hline $\mathbf{2 0 1 4}$ & 1.356 .030 & 236.252 \\
\hline $\mathbf{2 0 1 5}$ & 1.327 .085 & 236.075 \\
\hline $\mathbf{2 0 1 6}$ & 1.397 .338 & 234.454 \\
\hline
\end{tabular}

Fuente: Informes Anuales de la APBA

En 2013 (gráfico 1), destaca el mes de agosto con 312.017 pasajeros que realizan la ruta
Tarifa-Tánger Ville-Tarifa, coincidió la fiesta de fin del Ramadán en plena Operación Paso del Estrecho. En ese mismo año, se observan otros picos altos en octubre $(115.700$ viajeros aproximadamente) identificándose este aumento con la festividad Eid AlAdha (Fiesta del cordero); y en diciembre, explicándose este hecho por la existencia de periodos vacacionales correspondientes a celebraciones católicas como la Navidad en los países de residencia de los inmigrantes magrebíes. En 2014 se produjo un descenso del $7 \%$ en el número depasajerosque pasaron por el puerto de Tarifa, aunque mantuvo el máximo en el agosto (256.028 pasajeros), seguido del mes de julio, en que coincidieron la OPE y el fin de la festividad del Ramadán y en octubre, con la celebración de la pascua musulmana. En 2015, se dan los mismos supuestos anteriores, incrementándose el tráfico de pasajeros con respecto al 2014, aunque sin alcanzar los niveles del 2013. Se producen también índices elevados de tránsito de pasajeros, en julio (misma explicación que el año anterior) y en septiembre, mes de la fiesta del cordero. Por último, para 2016 se cumplen los mismos patrones anteriores, aunque desciende el número de pasajeros en agosto (en 2015, 258.258; y en 2016, 239.202). Se dan picos en los meses de abril (celebración de la pascua católica), septiembre (pascua musulmana) y julio (fiesta del Ramadán y OPE).

Para el número de vehículos (gráfico 2), se siguen las mismas pautas que en el gráfico 1 , es decir, el número de vehículos desciende a partir del 2014, pero produciéndose picos en los meses de verano. Este hecho se puede explicar también, por varios motivos como el aumento de la oferta de transporte (autobuses fundamentalmente) por parte de las navieras, incluyéndose en el precio del billete; y el incremento de la oferta de transporte público como trenes y autobuses durante los meses de mayor movimiento. 


\section{Gráfico 1. Viajeros de la ruta Tarifa-Tánger \\ Ville-Tarifa por meses, 2013-2016}

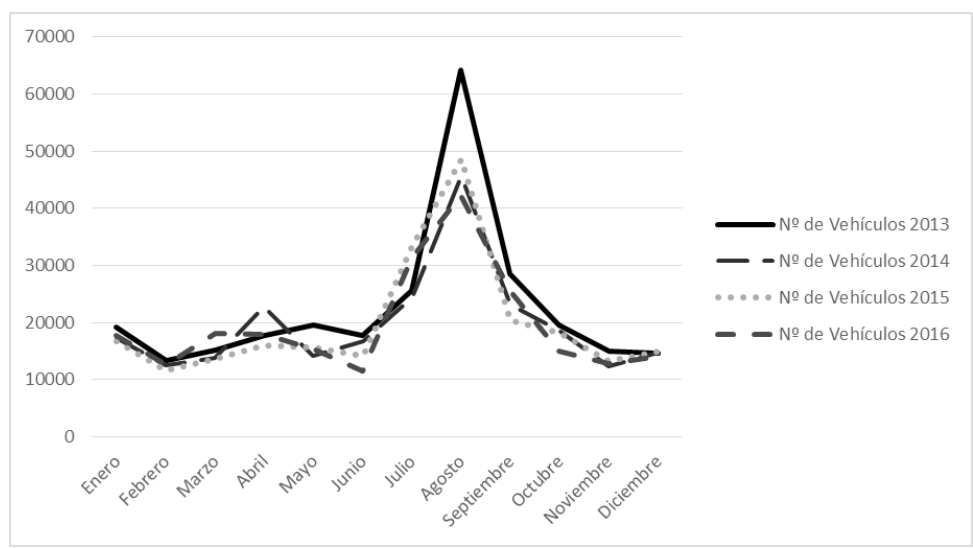

Fuente. Elaboración propia sobre datos de la APBA

\section{Gráfico 2. Vehículos en la ruta Tarifa-Tánger Ville-Tarifa por meses, 2013-2016}

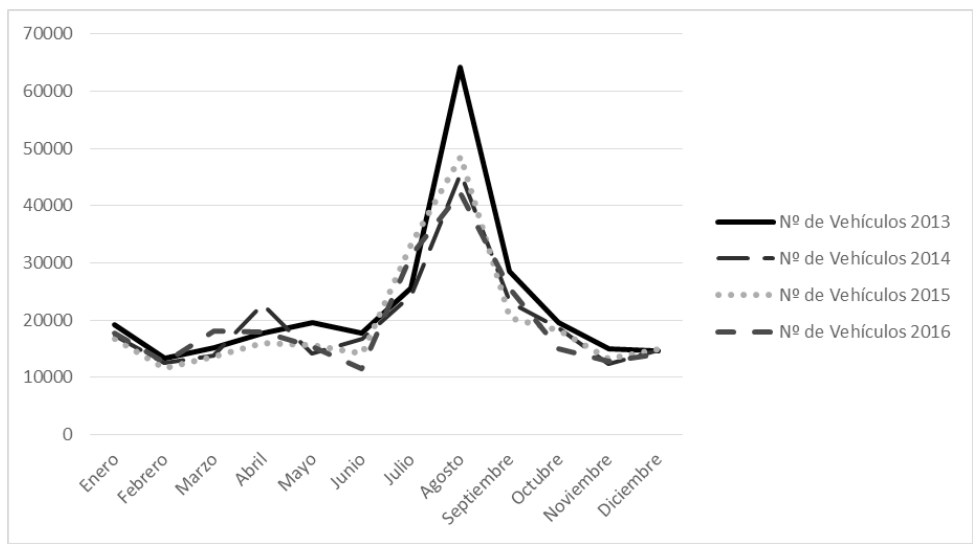

Fuente. Elaboración propia en base estadística APBA

El municipio de Tarifa, aunque tiene una población de alrededor de 18.000 habitantes, se ve triplicada durante los meses de verano (junio a septiembre) como consecuencia del turismo de sol y playa y deportivo y al movimiento migratorio de la OPE. Este último hecho lleva a una situación de colapso de las infraestructuras y los servicios de esta localidad, al encontrarse el puerto de Tarifa en el caso histórico de la ciudad.
Tarifa cuenta con una carretera nacional de tipo convencional (un carril en cada sentido, sin separación), la carretera $\mathrm{N}-340$, que conecta la población de su núcleo principal con la capital de la provincia (105 km hacia el noroeste) y con Algeciras (21 km al noreste). Los estados actuales de las infraestructuras viarias son deficientes y se encuentran en mal estado, en particular el tramo de Algeciras que es el más transitado durante la OPE. A 
esto se añade, los daños y movimientos de tierra causados por recientes temporales que, de no ser atendidos próximamente, representarán un serio problema en la OPE 2017.

Las plazas de aparcamiento del municipio asignadas por la APBA para la OPE alcanzaron en 2016, las 500 plazas; cifra escasa, si tenemos en cuenta el número de pasajeros magrebíes que llegan al municipio. Durante el período que dura la OPE se habilitan por parte del ayuntamiento explanadas fuera del casco antiguo para los autobuses y vehículos particulares.

El gasto municipal destinado a la OPE es incierto, puesto que el ayuntamiento no consigna una partida presupuestaria específica. A pesar de esto, para ayudar al buen funcionamiento de la OPE y con objeto de facilitar el tráfico de pasajeros y no se produzcan situaciones de colapso, el ayuntamiento destina parte de personal funcionarial (policías municipales y protección civil) a la gestión de la OPE.

\section{CONCLUSIONES}

La Operación Paso del Estrecho (OPE) se ha convertido en el segundo flujo migratorio musulmán más importante después de la peregrinación a la Meca. La organización y planificación que se hace por parte del gobierno de España resulta un éxito cada verano, incrementándose el tráfico de pasajeros y vehículos particulares cada año.

Esta operación lleva llamándose como tal desde 1991, habiéndose ejecutado planes anteriores desde 1986, que tenían la misma función que la actual OPE. Esta consiste en gestionar y planificar el movimiento migratorio que se produce durante los meses de verano como consecuencia del éxodo que realizan miles de inmigrantes magrebíes procedentes de Europa (fundamentalmente de Francia, Alemania y Bélgica) con el fin de visitar a sus familiares.

Más de dos millones y medio de personas cruzan el Estrecho de Gibraltar durante el periodo vacacional, eligiendo fundamentalmente los puertos gestionados por la Autoridad Portuaria Bahía de Algeciras (Algeciras y Tarifa) y Almería. Los puertos de Algeciras y Tarifa concentran el 75\% del tráfico total de viajeros y vehículos, siendo también durante el resto del año puertos con gran afluencia de pasajeros. Para el año 2016, más de 2,8 millones de personas viajaron durante la OPE por los puertos españoles y alrededor de 623.000 vehículos de particulares llevaron los buques a sus destinos. Estas cifras han ido incrementándose de forma conjunta en los últimos años, aumentando el tráfico en algunos puertos más que en otros.

Esta investigación se centra en buscar las implicaciones que tiene para el territorio de Tarifa una operación de gran envergadura como esta. Ya que, el municipio cuenta con una población aproximada de 18.000 habitantes, cifra que se ve acrecentada durante el verano por la OPE y por el turismo de sol y playa.

En el puerto de Tarifa se realiza una única ruta Tarifa-Tánger Ville-Tarifa, que concentra todo el tráfico de pasajeros y vehículos del puerto. Esta es la que miles de pasajeros durante el verano y el resto del año escogen para cruzar el Estrecho de Gibraltar. Durante los últimos cuatro años (desde el 2013 hasta el 2016) se ha notado un descenso en el tráfico de pasajeros (aunque sin variar los picos de afluencia durante los meses de verano), hecho que posiblemente esté relacionado con el 
colapso producido en los anteriores años y haya provocado que los viajeros escojan otras rutas; y por los temporales de vientos que implican el cierre del puerto (en el año 2016, el puerto de Tarifa cerró alrededor de 15 días según el boletín diario de la APBA).

Para finalizar, cabe señalar, que las infraestructuras que se posee el municipio no están acorde a las necesidades que se plantean en él. Las deficientes carreteras viarias de acceso y salida a Tarifa, la falta de aparcamiento y zona de descanso y la escasez de espacio en el puerto provocan que se congestione de tráfico el municipio y se perjudique tanto a su población como a los viajeros que desean cruzar el Estrecho de Gibraltar. Por tanto, el futuro de la OPE en el municipio de Tarifa pasa por mejorar la red viaria que la une con la capital y con Algeciras, las conexiones con otras ciudades del norte de África, ampliar la capacidad de estacionamiento y equipamiento del Puerto de Tarifa.

\section{REFERENCIAS BIBLIOGRÁFICAS}

Acosta Sánchez, M. (2016). Coopération et sécurité aux frontières extérieures européennes : le cas du détroit de Gibraltar. Annuarie français de droit international, 54, 197-223. http://doi.org/10.3406/ afdi.2008.4022

APBA (Autoridad Portuaria Bahía de Algeciras. (2015). Informe de gestión.

Bergmann, L. (2009). El arte rupestre paleolítico del extremo Sur de la Península Ibérica y la problemática de su conservación. Almoraima: revista de estudios campogibraltareños., 39, 45-65.

Boedec, G. Le. (2007). Le détroit de Gibraltar. EchoGeo, 2, 1-17.

Brand, L. (2006). Citizens abroad: Emigration and the state in the Middle East and North Africa. Cambridge University Press, 21, 1-18.

Cohen, A. (1995). Algunas reflexiones a propósito de la inmigración magrebí en España. Ería, 38, 287-302.

De Haas, H. (2008). The Myth of Invasion: the inconvenient realities of African migration to Europe. Third World Quarterly, 29(7), 1305-1322. http://doi.org/10.1080/01436590802386435

De Lera, D. (1995). La Inmigración en España a fines del siglo XX. Los que vienen a trabajar y los que vienen a descansar. Reis: revista de investigaciones sociológicas, 71, 225-245. http://doi. org/10.2307/40183869

Dirección General de Protección Civil y emergencias. (2014). Balance OPE 2014. Ministerio del Interior. Recuperado de http://www.interior.gob.es/prensa/noticias/-/asset_publisher/GHU8Ap6ztgsg/content/id/2509456

Dirección General de Protección Civil y emergencias. (2015). Balance OPE 2015. Ministerio del Interior. Recuperado de http://www.interior.gob.es/prensa/noticias/-/asset_publisher/GHU8Ap6ztgsg/content/id/4630880

Dirección General de Protección Civil y emergencias. (2016). Balance OPE 2016. Ministerio del Interior. Recuperado de http://www.interior.gob.es/prensa/noticias/-/asset_publisher/GHU8Ap6ztgsg/ content/id/6381934

Favell, A. (2008). The New Face of East-West Migration in Europe. Journal of Ethnic and Migration Studies, 34(5), 701-716. http://doi.org/10.1080/13691830802105947 
Fernández Alles, M. T., \& Fernández Alles, J. J. (2015). La Operación Paso del Estrecho. Gestión y Política Pública, XXIV (1), 51-84.

Finotelli, C. (2007). Italia, España y el modelo migratorio mediterráneo en el siglo XXI. Boletín Real Intituto Elcano.

Frolova, M. (2010). Los paisajes de la energia eólica: su percepción social y gestión en España. Nimbus, 25-26, 93-110.

Gauthier, C. (1997). Mobilités migratoires marocaines , sociabilités et échanges marchands. Revue européenne des migrations internationales, 13(3), 183-210. http://doi.org/10.3406/remi.1997.1572

Germond, B. (2005). De l'Atlantique à la Méditerranée vers une réorientation de la géostratégique navale dans l' espace euro-atlantique depues 1989. Cahiers de la Méditerranée, 71, 1-16. http:// doi.org/0395-9317

Ghatak, S., \& Sassoon, A. (2001). Migration and mobility: the European context. Springer.

Gómez Vallecillo, J. L. Estudio de las variables relacionadas con el estrés en la Operación Paso del Estrecho, Universidad de Cádiz 1-310 (2002).

Gozálvez Pérez, V. (1996). L' immigration étrangère en Espagne (1985-1994). Revue européenne des migrations internationales, 12(1), 11-38. http://doi.org/10.3406/remi.1996.1495

Grevemeyer, I., Matias, L., \& Silva, S. (2016). Mantle earthquakes beneath the South Iberia continental margin and Gulf of Cadiz? constraints from an onshore-offshore seismological network. Journal of Geodynamics, 99, 39-50. http://doi.org/10.1016/j.jog.2016.06.001

Heering, L., van der Erf, R., \& van Wissen, L. (2004). The role of family networks and migration culture in the continuation of Moroccan emigration: A gender perspective. Journal of Ethnic and Migration Studies, 30(2), 323-337. http://doi.org/10.1080/1369183042000200722

King, R. (2002). Towards a new map of European migration. International Journal of Population Geography, 8(2), 89-106. http://doi.org/10.1002/ijpg.246

La Moncloa. (2016). Plan de Verano 2016. Gobierno de España. Recuperado de http://www.lamoncloa.gob.es/consejodeministros/Paginas/enlaces/240616-enlaceverano.aspx

Lazaar, M. (1995). Gros Plan. La Migration internacionale Marocaine-aspects récents. Annuaire de l'Afrique du Nord, XXXIV, 994-1002.

López García, B. (1993). España. Puerta Europea del Magreb. Al-Andalus-Magreb, I, 123-143.

López García, B. (1996). La Operación Paso del Estrecho: tránsitos estacionales por la península. Atlas de la inmigración magrebí en España.

Marítima, S. de salvamento y seguridad. (2015). Informe Anual 2015.

Marte, A. (1996). Géodynamique de migrations internationals dans le monde. Canadian Journal of Political Science. Canadian Journal of Political Science, 1(29), 200-222.

Moreno Navarro, J. G., \& Ventura Fernández, J. (2007). Estrategias de desarrollo en el entorno del Estrecho de Gibraltar. La discontinuidad entre los arcos Atlánticos y Mediterráneo.

Moreno Pérez, P. (2012). Tarifa: Una alternativa turística. Aljaranda, 12-13. 
Mulero Mendigorri, A. (2001). Los espacios naturales protegidos en Andalucía: evolución, caracterización geográfica y singularidades. Ería, (54-55), 141-157.

Ouali, N. (2003). Les Marocaines en Europe: diversification des profils migratoires. Hommes et Migrations, (1242), 71-82.

Páez Granado, M. T., \& López García, B. (1991). La Emigración Marroquí en España: significación económica y sociocultural de su procedencia geográfica. AREAS: revista de Ciencias Sociales, 187-206.

Parejo Guerrero, E. (s. f.). La riqueza ornitológica del Campo de Gibraltar.

Román Lozano, A. (2008). Tarifa y el viento de levante. Aljaranda, 68, 1-3.

Sarriá Muñoz, A. (2016). La enseñanza en Tarifa en el siglo XVIII. Almoraima. Revista de Estudios Campogibraltareños, 45, 263-273.

Sempere Souvannavong, J. D. (2000). El tránsito de argelinos por el puerto de Alicante. Investigaciones Geográficas, (24), 111-130.

Soler, E., \& Vianello, A. (2006). La inalcanzable tercera <<M>>: visados y movilidad de personas en las relaciones euromediterráneas.

Streiff-Fénart, J., \& Poutignat, P. (2008). Nouadhibou «ville de transit»: Le rapport d'une ville à ses étrangers dans le contexte des politiques de contrôle des frontières de l'Europe. Revue européenne des migrations internationales, 24(2), 193-217. http://doi.org/10.4000/remi.4682

Tarifa, Ayuntamiento de (2016). Plan Económico-Financiero. Ayuntamiento de Tarifa.

\section{Para citaciones:}

Mañero-Viera, M., \& Segovia-Cuevas, M. C. (2017). La Operación Paso del Estrecho (OPE) en Cádiz (España). Panorama Económico, 25, 3, pp. 381-398.

\section{AUTORES}

Manuela Mañero-Viera

Doctorante en el Departamento de Economía General de la Universidad de Cádiz (España)

\section{María Concepción Segovia-Cuevas}

Profesora Titular e investigadora de la Universidad de Cádiz (España) en el Departamento de Economía General. Cuenta con un Ph.D. en Ciencias Económicas y Empresariales de la Universidad de Cadiz (España), con Maitrisse es Sciences-Demographie de la Universite de Montreal (Canda) y Licenciatura en Ciencias Económicas y Comerciales de la Universidad de Málaga (España). Sus líneas de investigación son: análisis sitémico, redes territoriales, demografía, población activa, empleo y desempleo, desarrollo y subdesarrollo. 


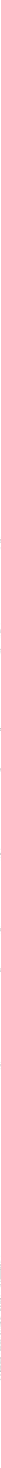

